\title{
A $L 1$ Minimization Optimal Corrective Explanation Procedure for Probabilistic Databases
}

\author{
Marco Baioletti@ and Andrea Capotorti ${ }^{(\varpi)}(1)$ \\ Dipartimento di Matematica ed Informatica, Università degli Studi di Perugia, \\ Perugia, Italy \\ \{marco.baioletti, andrea. capotorti\}@unipg.it \\ http://www.dmi.unipg.it
}

\begin{abstract}
We propose to use a, recently introduced, efficient $L 1$ distance minimization through mixed-integer linear programming for minimizing the number of valuations to be modified inside an incoherent probabilistic assessment. This is in line with one basic principle of optimal corrective explanation for decision makers.

A shrewd use of constraints and of slack variables permit to steer the correction of incoherent assessments towards aimed directions, like e.g. the minimal number of changes. Such corrective explanations can be searched alone, as minimal changes, or jointly with the property of being also inside the $L 1$ distance minimizers (in a bi-optimal point of view).

The detection of such bi-optimal solutions can be performed efficiently by profiting from the geometric characterization of the whole set of $L 1$ minimizers and from the properties of $L 1$ topology.
\end{abstract}

Keywords: Incoherence corrections $\cdot L 1$ constrained minimization • Mixed Integer Programming - Optimal corrective explanation • Probabilistic databases

\section{Introduction}

Uncertain data are nowadays becoming increasingly important in probabilistic databases [21] and they can emerge from traditional sources (e.g., by data integration like in [22]) or from the so called "next generation" sources (e.g., by information extraction like in [18]). Such kind of data bring with themselves a crucial characteristic: they must be consistent with sound uncertainty measures to be used properly, and this is not always assured, especially whenever they come from different sources of information.

As well outlined in [6], the way data fusion problem is tackled depends on the way information is represented. Since one of the most familiar and adopted

Supported by project "Algebraic statistics in a coherent setting for Bayesian networks" - DMI Unipg - Ricerca di Base 2018.

(C) Springer Nature Switzerland AG 2020

M.-J. Lesot et al. (Eds.): IPMU 2020, CCIS 1237, pp. 80-92, 2020.

https://doi.org/10.1007/978-3-030-50146-4_7 
measure of uncertainty is probability, consequently we focus here on the even more mandatory task of correcting inconsistent probabilistic databases (see, e.g., $[20])$.

The choice of correcting probability values reflects the willingness to maintain the probabilistic nature of the different sources of information. This because the agent who performs the fusion would preserve the expressive power of probability framework. Of course, a change of the uncertainty management paradigm could be possible by adopting more general degrees of belief to deal with ill-posed sentences, like e.g. Belief functions, Fuzzy Logic or possibility measures (there is a vast literature on this, see among the others $[1,5,7,8,10,16])$ but this would be a strong intervention on the information representation, with a possible loss in expressiveness (as defined in [16]), especially if the fusion process is performed by a "third party" with respect the original sources. Hence in the present contribution we describe a way to proceed when the fusion process is intended to follow probability rules.

Recently (see e.g. $[3,4]$ ), we have proposed an efficient method for correcting incoherent (i.e. inconsistent) probability assessments. This method is based on L1 distance minimization and Mixed Integer Programming (MIP) procedures can be designed to implement it, in line with what has been done in $[13,14]$ where such technique was introduced for the check of coherence problem.

The need of incoherence correction can be originated by different needs and can have different goals, hence we have designed specific MIP procedures to:

- correct straight unconditional assessments [4, §3];

- merge inconsistent databases $[4, \S 4]$;

- revise the belief in a dynamical setting $[3, \S 5]$;

- solve the so called plain statistical matching problem $[4, \S 6]$;

- solve its generalization of the statistical matching problem with missclassification errors $[9, \S 5]$;

- minimize the number of valuations to be modified, in line with one basic principle of optimal corrective explanation for decision makers (this contribution).

We are going to illustrate the last item of the previous list as it will appear in Sect. 4. Before doing it, we are going to recall in Sect. 2 the basic notions of partial probability assessments with the associated property of being coherent and in Sect. 3 how MIP is used to solve incoherence through $L 1$ distance minimization and to find the whole set of optimal solutions.

In Subsect. 4.1 the problem of finding all corrective explanations that are also at minimal $L 1$ distance from the initial assessment is posed and solved.

Two prototypical examples will illustrate our procedure.

\section{Probability Assessments}

In this section we recall some notions about probability assessments and coherence. 
Definition 1. A probability assessment is a tuple $\pi=(V, U, p, \mathfrak{C})$, where:

- $V=\left\{X_{1}, \ldots, X_{k}\right\}$ is a finite set of propositional variables, representing any potential event of interest;

- $U$ is a subset of $V$ of cardinality $n$ that contains the effective events taken into consideration;

- $p: U \rightarrow[0,1]^{n}$ is a vector which assigns a "potential" probability value $p\left(X_{i}\right)$, $i=1, \ldots, n$, to each variable in $U$;

- $\mathfrak{C}$ is a finite set of logical constraints which are present among all the variables in $V$ and that characterize the descriptions of the events of interest;

A probability assessment represents a state of knowledge about the probability for events in $U$ to be true. Such probability values can be evaluated (or "assessed", as more properly said into specific literature) on the base of observed data or of expert evaluations. Events are explicitly interconnected through the logical constraints $\mathfrak{C}$, which can be written involving all the potential events $V$, and not only those of $U$, to permit to extend an initial assessment to a larger domain without redefining the whole framework. The constraints $\mathfrak{C}$ are crucial to represent the domain of a database, especially whenever it is built by merging different sources of information, since they permit to represent any kind of compound event, i.e. any macro situation, as, for example, that an event is the conjunction of other two events, or the implications or incompatibilities among some elements of $V$.

As usually done in Boolean logic, $\mathfrak{C}$ can be expressed in conjunctive normal form (CNF), namely, $\mathfrak{C}=\left\{c_{1}, \ldots, c_{m}\right\}$ where each element $c_{i}$ of $\mathfrak{C}$ is a disjunctive logical clause of the form:

$$
c_{i}=\left(\bigvee_{h \in H_{i}} X_{h}\right) \vee\left(\bigvee_{l \in L_{i}} \neg X_{l}\right)
$$

with $X_{h}$ and $X_{l}$ in $V$, for some subsets of indexes $H_{i}, L_{i} \subseteq\{1, \ldots, k\}$.

This form results particularly helpful in the implementation part of the correction procedure, as we will described in the next section.

Since a probabilistic assessment $\pi$ is partial, i.e. not defined on a fully structured domain like a Boolean algebra, it may or not be coherent, that means consistent with a probability distribution. In literature there are different, but all equivalent, ways of defining coherence. They are based on semantic, syntactical or operational point of views (see, e.g., $[11,12,15,19]$ ), anyway a formulation in any approach can be easily translated into a formulation in a different one (see in particular the characterization results in $[11,12]$ ). For our proposal it is more fruitful to adopt the operational point of view already used in [2]. This will permit to face the coherence problem directly with mathematical programming tools.

Let us introduce now the notions of truth assignment and atom.

Definition 2. A truth assignment on $V$ is a function $\alpha: V \rightarrow\{0,1\}$. We denote by $2^{V}$ the set of all truth assignments. We denote with $\alpha \models \phi$ the fact 
that the assignment $\alpha \in 2^{V}$ satisfies a Boolean expression $\phi$ (which means that replacing each variable $x$ appearing in $\phi$ with the corresponding truth value $\alpha(x)$, the expression $\phi$ evaluates to 1$)$.

Definition 3. A truth assignment $\alpha \in 2^{V}$ is called atom for a probability assessment $\pi=(V, U, p, \mathfrak{C})$ if $\alpha$ satisfies all the logical constraints $c_{i} \in \mathfrak{C}$.

The coherence of a probability assessment is then defined as follows

Definition 4. A probability assessment $\pi=(V, U, p, \mathfrak{C})$ is coherent if there exists a probability distribution $\mu: 2^{V} \rightarrow[0,1]$ which satisfies the following properties

1. for each $\alpha \in 2^{V}$, if there exists a constraint $c_{i} \in \mathfrak{C}$ such that $\alpha \not \forall c_{i}$, then $\mu(\alpha)=0$

2. $\sum_{\alpha \in 2^{V}} \mu(\alpha)=1$;

3. for each $X_{j} \in U, \sum_{\alpha \in 2^{V}, \alpha \models X_{j}} \mu(\alpha)=p\left(X_{j}\right)$.

Checking the coherence of a probability assessment is a computational hard problem for which there exist many algorithmic approaches. In this paper we are mainly interested in the approach firstly described in [13], where the problem is solved by means of a Mixed Integer Programming (MIP) approach.

\section{Correcting Probability Assessments}

Whenever a probability assessment $\pi=(V, U, p, \mathfrak{C})$ results to not be coherent, it is possible to "modify" it in order to obtain a coherent probability assessment $\pi^{\prime}$ which is "as close as possible" to $\pi$.

Our approach consists in revising only the probability values $p$, because we consider the logical constraints $\mathfrak{C}$ as fixed. Hence the modified coherent assessment is $\pi^{\prime}=\left(V, U, p^{\prime}, \mathfrak{C}\right)$, with $p^{\prime}$ the corrected probability values.

Closeness between $p$ and $p^{\prime}$ is measured through a specific metric, and in our approach we are using the $L 1$ distance between $p$ and $p^{\prime}$, which is defined as

$$
d_{1}\left(p, p^{\prime}\right)=\sum_{i=1}^{n}\left|p\left(X_{i}\right)-p^{\prime}\left(X_{i}\right)\right| .
$$

The use of this distance has two important properties. First of all, minimization of the displacements $\left|p\left(X_{i}\right)-p^{\prime}\left(X_{i}\right)\right|$ respects the basic principle of minimal change in a numerical uncertainty setting. Secondly, there is a clear computational advantage with respect to other distances, like e.g. L2 or Kullback-Leibler divergence, because minimization of $L 1$ distance can be solved by MIP programming that has nowadays consolidated efficient algorithms implemented in several solvers while others distances would need less efficient and less robust algorithms for nonlinear (quadratic, logarithmic, etc.) problems. 
Given a probability assessment $\pi=(V, U, p, \mathfrak{C})$, we denote by $\mathcal{C}(\pi)$ the sets of all the $L 1$-corrections of $\pi$.

The procedure to find the corrections of $\pi=(V, U, p, \mathfrak{C})$ is described in [4]. Here, for sake of completeness, we recall the most important points. The basic idea is that in order to find the corrections of a probability assessment $\pi$ it is possible to solve a mixed integer program, which is denoted by $\mathcal{P} 1$. The method of obtaining $\mathcal{P} 1$ from $\pi$ exploits the same idea proposed in [13].

An important property is that if a probability assessment is coherent, there exists a sparse probability distribution $\mu$ so that $p^{\prime}$ can be written as a convex combination of at most $n+1$ atoms $\alpha^{(1)}, \ldots, \alpha^{(n+1)}$. Hence it is possible to build a MIP problem $\mathcal{P} 1$, whose size is polynomial with respect to the size of $\pi$.

The non-negative variables for $\mathcal{P} 1$ are summarized in Table 1.

Table 1. List of variables in the MIP program $\mathcal{P} 1$ used to correct an incoherent probabilistic assessments.

\begin{tabular}{l|l|l|l}
\hline Name & Indexes & Size & Type \\
\hline \multirow{2}{*}{$a_{i j}$} & $i=1, \ldots, n$ & & \\
& $j=1, \ldots, n+1$ & $n(n+1)$ & Binary \\
\hline & $i=1, \ldots, n$ & & \\
$b_{i j}$ & & $n(n+1)$ & Real \\
& $j=1, \ldots, n+1$ & & \\
\hline$q_{j}$ & $j=1, \ldots, n+1$ & $n+1$ & Real \\
\hline$r_{i}$ & $i=1, \ldots, n$ & $n$ & Real \\
\hline$s_{i}$ & $i=1, \ldots, n$ & $n$ & Real \\
\hline
\end{tabular}

The linear constraints of $\mathcal{P} 1$ are

- for $i=1, \ldots, m$ and $j=1, \ldots, n+1$

$$
\sum_{h \in H_{i}} a_{h, j}+\sum_{l \in L_{i}}\left(1-a_{l, j}\right) \geq 1
$$

- for each $i=1, \ldots, n$,

$$
\sum_{j=1}^{n+1} b_{i j}=p\left(X_{i}\right)+\left(r_{i}-s_{i}\right)
$$

- for $i=1, \ldots, n$ and $j=1, \ldots, n+1$,

$$
0 \leq b_{i j} \leq a_{i j}, \quad a_{i j}-1+q_{j} \leq b_{i j} \leq q_{j}
$$




$$
\sum_{i=1}^{n+1} q_{j}=1
$$

- for $i=1, \ldots, n$,

$$
r_{i} \leq 1, \quad s_{i} \leq 1
$$

where, we recall, $n$ is the cardinality of $p, m$ the cardinality of $\mathfrak{C}$, while $H_{i}$ and $L_{i}$ are the indexes subsets in the disjunctive normal form (1) of each $c_{i} \in \mathfrak{C}$.

The variables $a_{i j}$ are binary, i.e. constrained in $\{0,1\}$. Each value $a_{i j}$ corresponds to the atom component $\alpha^{(j)}\left(X_{i}\right)$, for $i=1, \ldots, n$ and $j=1, \ldots, n+1$. Indeed, the constraint (3)

$$
\begin{array}{rl}
\sum_{h \in H_{i}} a_{h j}+\sum_{l \in L_{i}}\left(1-a_{l j}\right) \geq 1 & i=1, \ldots, m \\
j & =1, \ldots, n+1
\end{array}
$$

forces each atom $\alpha^{(j)}\left(a_{1 j}, \ldots, a_{n j}\right)$ in the solution to satisfy all the clauses $c_{i} \in \mathfrak{C}$.

The values $q_{1}, \ldots, q_{n+1}$ represent the coefficient of the convex combination which generates $p^{\prime}$, which also correspond to the probabilities $\mu\left(\alpha^{(1)}\right), \ldots$, $\mu\left(\alpha^{(n+1)}\right)$.

The constraint (3)

$$
\begin{aligned}
0 \leq b_{i j} \leq a_{i j}, \quad a_{i j}-1+q_{j} \leq b_{i j} \leq q_{j} \quad & i=1, \ldots, n \\
j & =1, \ldots, n+1
\end{aligned}
$$

is equivalent to the non linear constraint

$$
b_{i j}=a_{i j} \cdot q_{j} \text { for } i=1, \ldots, n \text { and } j=1, \ldots, n+1 .
$$

Since $a_{i j}=1$ if and only if $\alpha^{(j)}$ satisfies $\mathbf{X}_{\mathbf{i}}$, the sum

$$
\sum_{j=1}^{n+1} b_{i j}
$$

is also equal to $\mathbf{p}^{\prime}\left(\mathbf{X}_{\mathbf{i}}\right)$.

The variables $r_{i}, s_{i}$ are slack variables, which represent the positive and the negative difference between $p\left(X_{i}\right)$ and $p^{\prime}\left(X_{i}\right)$, that permit to translate the inequalities $p^{\prime}\left(X_{i}\right) \leq p\left(X_{i}\right) p^{\prime}\left(X_{i}\right) \geq p\left(X_{i}\right)$ into equations (4):

$$
\sum_{j=1}^{n+1} b_{i j}=p\left(X_{i}\right)+\left(r_{i}-s_{i}\right) i=1, \ldots, n
$$

Finally, the program $\mathcal{P} 1$ is composed by the constraints $(1-5)$ and its objective function to be minimized is

$$
\sum_{i=1}^{n}\left(r_{i}+s_{i}\right)
$$


It is possible to prove that any solution of the linear program $\mathcal{P} 1$ corresponds to a $L 1$-correction $p^{\prime}$ of $p[4]$.

In many situations $\mathcal{C}(\pi)$ has more than one element and the MIP problem is able to find just one solution, which could not be a good representative of all the elements of $\mathcal{C}(\pi)$, as happens when it is an extreme value.

Hence program $\mathcal{P} \mathbf{1}$ must be associated to an other MIP program $\mathcal{P} \mathbf{2}$ to generate the most "baricentric" point $\bar{p}$ of $\mathcal{C}(\pi)$, i.e. a solution that spreads as much uniformly as possible the numerical amount of the distance (2) amongst the $n$ values in $p$ (for details and formal proofs refer again to [4]).

In $\mathcal{P} 2$ all the constraints and the variables of $\mathcal{P} 1$ are reported and it contains a new real variable $z$, which is subject to the constraints $r_{i}+s_{i} \leq z$, for $i=1, \ldots, n$ (hence $z \geq \max _{i=1, \ldots, n}\left(r_{i}+s_{i}\right)$ ), and the new additional constraint $\sum_{i=1}^{n}\left(r_{i}+s_{i}\right)=\delta$, where $\delta$ is the optimal value of the objective function in $\mathcal{P} 1$.

In this way, the $\mathcal{P} 2$ objective function to be minimized is simply $z$.

Using $\bar{p}$, it is possible to find the face $F_{1}$ of the polytope of coherent assessments $\mathcal{Q}$ where $\mathcal{C}(\pi)$ lies.

The face $F_{1}$ is itself a convex set with at most $n+1$ atoms as extremal points, which can be found as a part of the solutions of $\mathcal{P} 2$ (i.e., the optimal values of $\left.a_{i j}\right)$.

By looking at the signs of $\bar{p}\left(X_{i}\right)-p\left(X_{i}\right)$, for $i=1, \ldots, n$, it is also possible to determine the face $F_{2}$ of the $L 1$ ball $\mathcal{B}_{\pi}(\delta)$ which contains $\mathcal{C}(\pi)$. Indeed, $F_{2}$ is a convex set with at most $n$ extremal points of the form

$$
p+\operatorname{sign}\left(\bar{p}\left(X_{j}\right)-p\left(X_{j}\right)\right) \cdot \delta \cdot e_{j}
$$

The whole set of corrections will result as:

$$
\mathcal{C}(\pi)=F_{1} \cap F_{2}
$$

The computation of (10) can be done by using a procedure FaceEnum, which, given a polyhedron, finds its $\mathrm{H}$ representation formed by the half-spaces that contain its faces, and a procedure VertexEnum, which finds the V representation constituted by its extreme points or vertices. Examples of FaceEnum and VertexEnum are described, e.g., in [17].

The overall procedure is described in the following pseudo-code.

\section{procedure Correct}

Input: assessment $(V, U, p, \mathfrak{C})$

Output: extremal points $W$ and minimum distance $\delta$

\section{begin}

prepare MIP program $\mathcal{P} 1$

solve $\mathcal{P} 1$

extract the optimal value $\delta$

if $\delta=0$ then

return $(\{p\}, 0)$

else 
prepare MIP program $\mathcal{P} 2$

solve $\mathcal{P} 2$

extract the values $a_{i j}, r_{i}, s_{i}$

$E_{1}:=$ columns of matrix $a_{i j}$

compute $\bar{p}$ from $r_{i}, s_{i}$

compute $E_{2}$ with Formula (9)

$H_{1}:=\operatorname{FaceEnum}\left(E_{1}\right)$

$H_{2}:=\operatorname{FaceEnum}\left(E_{2}\right)$

$Q:=\operatorname{VertexEnum}\left(H_{1} \cup H_{2}\right)$

endif

return $(Q, \delta)$

end

\section{Optimal Corrective Explanation}

The approach to correcting probability assessments described in the previous section takes into account only the sum of the corrections made on the probability values.

If we consider that enforcing the coherence of a probability assessment can be seen as a process of constraint satisfaction, a different perspective can be used. Indeed, as in an interactive tasks of constraint programming, it could be helpful to detect a minimal number of events whose probability value should be modified. In this way, the correction needed to obtain a coherent assessment has a better explanation, because the modifications are concentrated to a small number of events, instead being spread on more events. In any case, also the sum of the corrections must be minimal.

Concretely, the objective function can take into account of both: the number of the affected probability values and the sum of all the modifications. A procedure to compute this result is based on the previously described procedure Correct, with some simple modifications.

First of all, the program $\mathcal{P} 1$ is extended by adding specific variables and constraints that permit to "count" the modified probability values.

The new integer variables are $I_{i}$, for $i=1, \ldots, n$, while the additional constraints are

$$
r_{i}+s_{i} \leq I_{i} \leq 1, \quad i=1, \ldots, n
$$

If we just search for a solution with a minimal number of changes, the new objective function is simply

$$
\sum_{i=1}^{n} I_{i} .
$$

Let us show its application with a simple numerical example:

Example 1. Let $U=V=\left\{X_{1}, X_{2}, X_{3}\right\}$

$\mathfrak{C}=\left(X_{2} \vee X_{3}\right) \wedge\left(X_{2} \vee \neg X_{1}\right) \wedge\left(X_{3} \vee \neg X_{1}\right)$ 
$p=(.9, .8, .9)$

hence with $n=3, m=3$ and $H_{1}=\{2,3\}, L_{1}=\emptyset, H_{2}=\{2\}, L_{2}=\{1\}$, $H_{3}=\{3\} L_{3}=\{1\}$.

By plugging these values into constraints (3) - (7),(11) and by searching the optimal minimal value of the objective function (12) we obtain an optimal corrective explanation of $I_{2}=1$ with $r_{2}=.2$. Hence only $p_{2}$ must be modified into $p_{2}^{\prime}=1.0$.

Note that this correction actually minimize also the L1 distance objective function (8).

If, on the contrary, we start from the incoherent assessment $p=(.99, .8, .9)$, we obtain as optimal corrective explanation $I_{2}=1$ and $I_{3}=1$, with $r_{2}=.2$ and $r_{3}=.09$, so that $p_{2}^{\prime}=1.0$ and $p_{3}^{\prime}=.99$. Note that also this correction minimize the $L 1$ distance objective function.

\subsection{Optimal Corrective Explanation at Minimal L1 Distance}

From the previous example one could be tempted to induce that each optimal corrective explanation is also a $L 1$ distance minimizer, but obviously it is not always the case since the polytope of coherent assessments $\mathcal{Q}$ could have inner points in the same direction $p \pm \delta \cdot\left(I_{1}, \ldots, I_{n}\right)$.

To be sure to find on optimal corrective explanation that has also the minimal $L 1$ distance, it is enough to change only the objective function to

$$
\sum_{i=1}^{n} r_{i}+s_{i}+I_{i} .
$$

Is is immediate to prove the following

Proposition 1. Any assessment $p^{\prime}$ induced by the solution of the MIP program with objective function (13) has a minimal number of corrections and a minimal L1 distance w.r.t. the initial assessment $p$.

In fact, if there exists another correction $p^{\prime \prime}$ with the same number of changed components but with a smaller $L 1$ distance, or with the same distance but with a smaller number of changed components, the objective function (13) would inevitably result smaller since the constraints that make the $I_{i}$ equal to 1 whenever any one of the correction components $r_{i}$ or $s_{i}$ is different from zero.

Let us see an other toy example that shows what we can obtain.

Example 2. $U=V=\left\{X_{1}, X_{2}, X_{3}\right\}$

$\mathfrak{C}=\left(X_{2} \vee X_{3} \vee \neg X_{1}\right) \wedge\left(X_{3} \vee \neg X_{1} \vee \neg X_{2}\right) \wedge\left(\neg X_{1} \vee \neg X_{2} \vee \neg X_{3}\right) \wedge\left(X_{1} \vee X_{3} \vee \neg X_{2}\right)$

$p=(1.0, .3, .2)$

that could lead to an optimal corrective explanation of $I_{1}=1$ and $I_{2}=1$ with 
$s_{1}=1.0$ and $s_{2}=.1$ hence $p_{1}$ must be modified into $p_{1}^{\prime}=0.0$ and $p_{2}$ must be modified into $p_{2}^{\prime}=0.2$. Also this correction minimizes the L1 distance objective function (2) with

$$
d_{1}\left(p, p^{\prime}\right)=1.1
$$

If we want to collect all such corrections that simultaneously minimize the number of changes and the $L 1$ distance, let us denote such optimal corrections by $\mathcal{O}(\pi)$, we can resort to the reasoning illustrated in the previous section to find the whole set of minimal distance corrections $\mathcal{C}(\pi)$.

Since $\mathcal{C}(\pi)$ has been obtained in (10) as the intersection of the two faces $F_{1}$ and $F_{2}$, if it contains some extremal points of the face $F_{2}$, that we recall is a face of the $L 1$ ball $\mathcal{B}_{\pi}(\delta)$, then these are the searched solutions. In fact, since any other correction $p^{\prime \prime}$ is a convex combination of such extremal points can have only a greater number of changes w.r.t. the extremes. In this case $\mathcal{O}(\pi)$ is composed of isolated points and has a cardinality of at most $n$.

If, on the contrary, $\mathcal{C}(\pi)$ does not contain any extremal point of $F_{2}$, the extremal points of the convex set $F_{1} \cap F_{2}$ surely belong to the searched solutions $\mathcal{O}(\pi)$ as long as any other convex combination among those that change the same values. No other point in $\mathcal{C}(\pi)$ can belong to $\mathcal{O}(\pi)$, since it will result as convex combination of extremal points that changes different components and hence it will change the union of those. In such a case $\mathcal{O}(\pi)$ will result as some isolated vertex and some edges of $F_{1} \cap F_{2}$.

Let us show the two different situations by referring to the previous introduced toy examples.

In Example 1 we have (see Fig. 1 ) that $\mathcal{C}(\pi)=F_{1} \cap F_{2}$ it has four extremal points $p^{\prime}=(.9,1.0, .9), p^{\prime \prime}=(.7, .8, .9), p^{\prime \prime \prime}=(.8, .8,1.0), p^{i v}=(.9, .9,1.0)$, and contains two extremal points of $F_{2}$ so that $\mathcal{O}(\pi)$ is the isolated vertexes $p$ and $p^{\prime \prime}$.

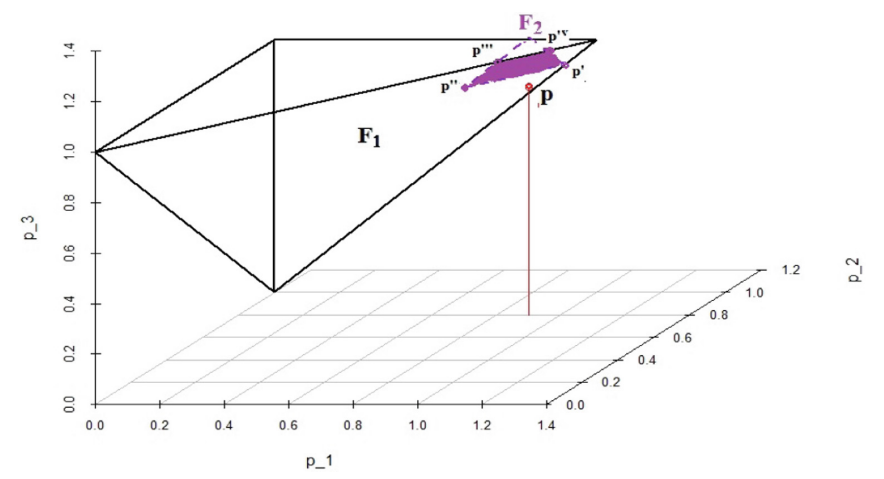

Fig. 1. Whole sets of corrections for Ex.1: $\mathcal{C}(\pi)$ is the colored area of $F_{1} \cap F_{2}$ with extremal points $\left\{p^{\prime}, p^{\prime \prime}, p^{\prime \prime \prime}, p^{i v}\right\}$; while $\mathcal{O}(\pi)$ is the two separated points $\left\{p^{\prime}, p^{\prime \prime}\right\}$ (Color figure online) 
In Example 2 we have (see Fig. 2) that $\mathcal{C}(\pi)=F_{1} \cap F_{2}$ does not contain any extremal point of $F_{2}$ and it has five extremal points $p^{\prime}=(0.0, .2, .2), p^{\prime \prime}=$ $(.2,0.0, .2), p^{\prime \prime \prime}=(1.0,0.0,1.0), p^{i v}=(.7, .3,1.0), p^{v}=(0.0, .3, .3)$; while $\mathcal{O}(\pi)$ is the isolated vertex $p^{\prime \prime \prime}$ and the two edges $\overline{p^{\prime} p^{\prime \prime}}$, and $\overline{p^{i v} p^{v}}$.

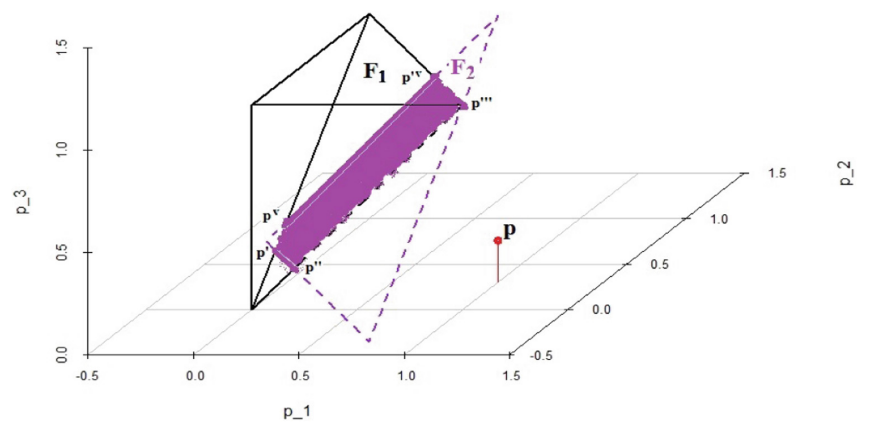

Fig. 2. Whole sets of corrections for Ex.2: $\mathcal{C}(\pi)$ is the colored area of $F_{1} \cap F_{2}$ with extremal points $\left\{p^{\prime}, p^{\prime \prime}, p^{\prime \prime \prime}, p^{i v}, p^{v}\right\}$; while $\mathcal{O}(\pi)$ is $p^{\prime \prime \prime}$ and the two solid edges $\overline{p^{\prime} p^{\prime \prime}}$, and $\overline{p^{i v} p^{v}}$ (Color figure online)

\section{Conclusion}

With this contribution we have shown how Mixed Integer Programming can be profitable used to solve probabilistic databases conflicts.

In particular, using additional variables and constraints, it is possible to render linear most practical problems. Specifically, we focused on the problem of minimizing the number of corrections in an incoherent set of probability assessments, obtaining a so called optimal corrective explanation. A smart use of the involved slack variables and an immediate modification of the objective function has easily permitted to obtain a double goal: minimal number of changes at a minimal $L 1$ distance, in a bi-objective prospective.

By profiting from geometric properties of the whole set of minimal $L 1$ distance solutions, jointly with the topology of the $L 1$ metric, it was possible to characterize the whole set of such bi-optimal solutions so that we easily proposed an efficient procedure to compute them. The knowledge of all the bi-optimal corrective explanations permits the decision maker to select the most appropriate adjustment of an inconsistent probabilistic database.

In next future we are going to implement the proposed method in a unique procedure in order to deal and to solve real practical problems of reasonable size. 


\section{References}

1. Bacchus, F., Grove, A.J., Halpern, J.Y., Koller, D.: From statistical knowledge bases to degrees of belief. Artif. Intell. 87(1-2), 75-143 (1996). https://doi.org/10. 1016/S0004-3702(96)00003-3

2. Baioletti, M., Capotorti, A., Tulipani, S., Vantaggi, B.: Elimination of Boolean variables for probabilistic coherence. Soft Comput. 4(2), 81-88 (2000). https:// doi.org/10.1007/s005000000040

3. Baioletti, M., Capotorti, A.: Efficient L1-based probability assessments correction: algorithms and applications to belief merging and revision. In: Proceedings of the 9th International Symposium on Imprecise Probability: Theories and Applications (ISIPTA 2015), Pescara (IT), pp. 37-46. ARACNE (2015)

4. Baioletti, M., Capotorti, A.: A $L 1$ based probabilistic merging algorithm and its application to statistical matching. Appl. Intell. 49(1), 112-124 (2018). https:// doi.org/10.1007/s10489-018-1233-z

5. Benferhat, S., Dubois, D., Prade, H.: How to infer from inconsistent beliefs without revising? In: Proceedings of the 14th International Joint Conference on Artificial Intelligence (IJCAI 1995), Montréal, Canada, 20-25 August, pp. 1449-1455 (1995)

6. Benferhat, S., Dubois, D., Prade, H.: From semantic to syntactic approaches to information combination in possibilistic logic. In: Bouchon-Meunier, B. (eds) Aggregation and Fusion of Imperfect Information, pp. 141-161. Physica-Verlag HD (1998). https://doi.org/10.1007/978-3-7908-1889-5_9

7. Benferhat, S., Sossai, C.: Reasoning with multiple-source information in a possibilistic logic framework. Inf. Fusion 7, 80-96 (2006). https://doi.org/10.1016/j. inffus.2005.01.006

8. Bosc, P., Pivert, O.: Querying possibilistic databases: three interpretations. In: Yager, R., Abbasov, A., Reformat, M., Shahbazova, S. (eds.) Soft Computing: State of the Art Theory and Novel Applications. STUDFUZZ, vol. 291, pp. 161176. Springer, Heidelberg (2013). https://doi.org/10.1007/978-3-642-34922-5_12

9. Buonunori, G., Capotorti, A.: Behavior of $L_{1}$-based probabilistic correction applied to statistical matching with misclassification information. In: Kratochvíl, V., Vejnarová, J. (eds.) Proceedings of the 11th Workshop on Uncertainty Processing (WUPES 2018), Trebon, Czech Republic, pp. 25-36. MatfyzPress, Czech Republic (2018)

10. Castro, J.L., Herrera, F., Verdegay, J.L.: Knowledge-based systems and fuzzy boolean programming. Intell. Syst. 9(2), 211-225 (1994). https://doi.org/10.1002/ int. 4550090203

11. Coletti, G.: Coherent numerical and Ordinal probabilistic assessments. IEEE Trans. Syst. Man Cybern. 24, 1747-1754 (1994). https://doi.org/10.1109/21. 328932

12. Coletti, G., Scozzafava, R.: Probabilistic Logic in a Coherent Setting. Kluwer, Series "Trends in Logic", Dordrecht (2002). https://doi.org/10.1007/978-94-0100474-9

13. Cozman, F.G., di Ianni, L.F.: Probabilistic satisfiability and coherence checking through integer programming. In: van der Gaag, L.C. (ed.) ECSQARU 2013. LNCS (LNAI), vol. 7958, pp. 145-156. Springer, Heidelberg (2013). https://doi.org/10. 1007/978-3-642-39091-3_13

14. Cozman, F.G., di Ianni, L.F.: Probabilistic satisfiability and coherence checking through integer programming. Int. J. Approximate Reason. 58, 57-70 (2015). https://doi.org/10.1016/j.ijar.2014.09.002 
15. de Finetti, B.: Teoria della Probabilità. Torino Einaudi, 1970. Wiley, London (1974). https://doi.org/10.1002/9781119286387. (English translation Theory of probability)

16. Dubois, D., Liu, W., Ma, J., Prade, H.: The basic principles of uncertain information fusion. An organised review of merging rules in different representation frameworks. Inf. Fusion Part A 32, 12-39 (2016). https://doi.org/10.1016/j.inffus. 2016.02.006

17. Fukuda, K.: Lecture: Polyhedral Computation, Spring (2011). Institute for Operations Research and Institute of Theoretical Computer Science, ETH Zurich. https://inf.ethz.ch/personal/fukudak/lect/pclect/notes2015/PolyComp2015.pdf

18. Gupta, R., Sarawagi, S.: Creating probabilistic databases from information extraction models. In: Proceedings of the 32nd International Conference on Very Large Data Bases, pp. 965-976 (2006)

19. Lad, F.: Operational Subjective Statistical Methods: A Mathematical, Philosophical, and Historical Introduction. Wiley, New York (1996)

20. Lian, A.X., Chen, A.L., Song, A.S.: Consistent query answers in inconsistent probabilistic databases. In: Proceedings of the 2010 ACM SIGMOD International Conference on Management of Data, Indianapolis, Indiana, pp. 303-314 (2010). https:// doi.org/10.1145/1807167.1807202

21. Suciu, D., Olteanu, D., Ré, C., Koch, C.: Probabilistic Databases, Synthesis Lectures On Data Management. Morgan \& Claypool Publishers (2011). https://doi. org/10.2200/S00362ED1V01Y201105DTM016

22. Dong, X.L., Halevy, A., Yu, C.: Data integration with uncertainty. Very Large Data Bases J. 18, 469-500 (2009). https://doi.org/10.1007/s00778-008-0119-9 